\title{
Erratum to: The Appendicitis Inflammatory Response Score: A Tool for the Diagnosis of Acute Appendicitis that Outperforms the Alvarado Score
}

Manne Andersson • Roland E. Andersson

Published online: 12 June 2012

(c) Société Internationale de Chirurgie 2012

Erratum to: World J Surg (2008) 32:1843-1849

DOI 10.1007/s00268-008-9649-y

In the original article, the CRP concentration measurements are incorrect in Tables 2 and 7. Following are the corrected tables:

The online version of the original article can be found under doi:10.1007/s00268-008-9649-y.

M. Andersson $(\bowtie) \cdot R$. E. Andersson

Department of Surgery, County Hospital Ryhov,

55185 Jönköping, Sweden

e-mail: manne.andersson@lj.se

R. E. Andersson

Department of Surgery, University Hospital, 58185 Linköping,

Sweden
Table 2 The new scores and the Alvarado score for comparison

\begin{tabular}{|c|c|c|c|}
\hline \multirow[t]{2}{*}{ Score } & \multicolumn{3}{|c|}{ Proposed score } \\
\hline & Regression & Simplified & Alvarado \\
\hline Relocation of pain & ns & - & +1 \\
\hline Vomiting & 0.45 & +1 & +1 \\
\hline Pain in RIF & 1.12 & +1 & +2 \\
\hline Anorexia & ns & - & +1 \\
\hline Male gender & ns & - & - \\
\hline \multicolumn{4}{|c|}{ Rebound tenderness or muscular defense } \\
\hline None & 0 & 0 & 0 \\
\hline Light & 1.54 & +1 & +1 \\
\hline Medium & 1.90 & +2 & +1 \\
\hline Strong & 2.32 & +3 & +1 \\
\hline \multicolumn{4}{|l|}{ Body temperature } \\
\hline $37.5-37.9^{\circ} \mathrm{C}$ & 0 & 0 & +1 \\
\hline $38.0-38.4^{\circ} \mathrm{C}$ & 0 & 0 & +1 \\
\hline$\geq 38.5^{\circ} \mathrm{C}$ & 0.85 & +1 & +1 \\
\hline \multicolumn{4}{|c|}{ Proportion polymorphonuclear leukocytes } \\
\hline $70-74 \%$ & 0.92 & +1 & 0 \\
\hline $75-84 \%$ & 0.92 & +1 & +1 \\
\hline$\geq 85 \%$ & 1.41 & +2 & +1 \\
\hline \multicolumn{4}{|l|}{ WBC count } \\
\hline $10.0-14.9 \times 10^{9} / \mathrm{L}$ & 0.96 & +1 & +2 \\
\hline$\geq 15.0 \times 10^{9} / \mathrm{L}$ & 1.46 & +2 & +2 \\
\hline \multicolumn{4}{|l|}{ CRP concentration } \\
\hline $10-49 \mathrm{mg} / \mathrm{L}$ & 1.04 & +1 & - \\
\hline$\geq 50 \mathrm{mg} / \mathrm{L}$ & 2.35 & +2 & - \\
\hline
\end{tabular}


Table 7 Scoring sheet with proposed clinical algorithm

\begin{tabular}{lll}
\hline Vomiting & & 1 \\
$\begin{array}{l}\text { Pain in right inferior fossa } \\
\begin{array}{l}\text { Rebound tenderness or } \\
\text { muscular defense }\end{array}\end{array}$ & Light & 1 \\
& Medium & 1 \\
& Strong & 2 \\
Body temperature $\geq 38.5$ & & 3 \\
Polymorphonuclear leukocytes & $70-84 \%$ & 1 \\
& $\geq 85 \%$ & 1 \\
WBC count & $10.0-14.9 \times 10^{9} / \mathrm{L}$ & 1 \\
& $\geq 15.0 \times 10^{9} / \mathrm{L}$ & 2 \\
CRP concentration & $10-49 \mathrm{mg} / \mathrm{L}$ & 1 \\
& $\geq 50 \mathrm{mg} / \mathrm{L}$ & 2 \\
Sum $(0-12)$ & &
\end{tabular}

$(0-12)$

Sum $0-4=$ Low probability. Outpatient follow-up if unaltered general condition

Sum 5-8 = Indeterminate group. In-hospital active observation with rescoring/imaging or diagnostic laparoscopy according to local traditions

Sum 9-12 = High probability. Surgical exploration is proposed 